\title{
Non-equilibrium surface tension of the vapour-liquid interface of active Lennard- Jones particles
}

Siddharth Paliwal, Vasileios Prymidis, Laura Filion, and Marjolein Dijkstra

Citation: The Journal of Chemical Physics 147, 084902 (2017); doi: 10.1063/1.4989764

View online: http://dx.doi.org/10.1063/1.4989764

View Table of Contents: http://aip.scitation.org/toc/jcp/147/8

Published by the American Institute of Physics

\section{Articles you may be interested in}

Contact angles from Young's equation in molecular dynamics simulations

The Journal of Chemical Physics 147, 084708 (2017); 10.1063/1.4994088

Perspective: Surface freezing in water: A nexus of experiments and simulations

The Journal of Chemical Physics 147, 060901 (2017); 10.1063/1.4985879

Molecular dynamics study of the potential of mean force of SDS aggregates

The Journal of Chemical Physics 147, 084903 (2017); 10.1063/1.4998549

Drying and wetting transitions of a Lennard-Jones fluid: Simulations and density functional theory

The Journal of Chemical Physics 147, 044701 (2017); 10.1063/1.4993515

Novel tilt-curvature coupling in lipid membranes

The Journal of Chemical Physics 147, 084702 (2017); 10.1063/1.4990404

Pair 2-electron reduced density matrix theory using localized orbitals

The Journal of Chemical Physics 147, 084101 (2017); 10.1063/1.4999423

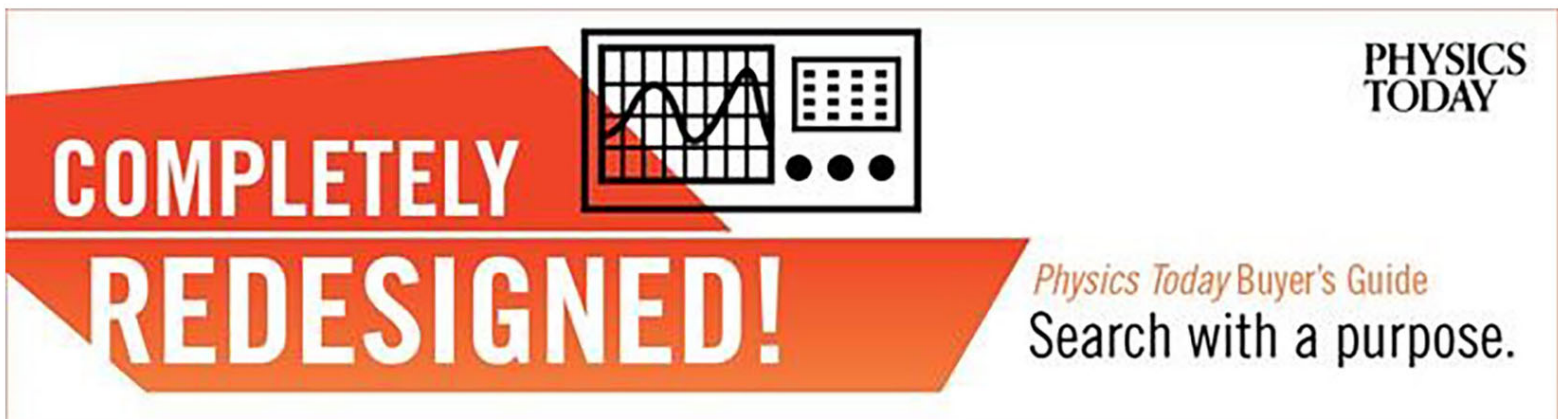




\title{
Non-equilibrium surface tension of the vapour-liquid interface of active Lennard-Jones particles
}

\author{
Siddharth Paliwal, ${ }^{\mathrm{a}, \mathrm{b})}$ Vasileios Prymidis, ${ }^{\text {b) }}$ Laura Filion, and Marjolein Dijkstra ${ }^{\mathrm{c})}$ \\ Soft Condensed Matter, Debye Institute for Nanomaterials Science, Utrecht University, Princetonplein 5 , \\ 3584 CC Utrecht, The Netherlands
}

(Received 12 June 2017; accepted 5 August 2017; published online 23 August 2017)

\begin{abstract}
We study a three-dimensional system of self-propelled Brownian particles interacting via the LennardJones potential. Using Brownian dynamics simulations in an elongated simulation box, we investigate the steady states of vapour-liquid phase coexistence of active Lennard-Jones particles with planar interfaces. We measure the normal and tangential components of the pressure tensor along the direction perpendicular to the interface and verify mechanical equilibrium of the two coexisting phases. In addition, we determine the non-equilibrium interfacial tension by integrating the difference of the normal and tangential components of the pressure tensor and show that the surface tension as a function of strength of particle attractions is well fitted by simple power laws. Finally, we measure the interfacial stiffness using capillary wave theory and the equipartition theorem and find a simple linear relation between surface tension and interfacial stiffness with a proportionality constant characterized by an effective temperature. Published by AIP Publishing. [http://dx.doi.org/10.1063/1.4989764]
\end{abstract}

\section{INTRODUCTION}

Many-particle systems that are driven out-of-equilibrium exhibit intriguing collective behavior like clustering, laning, swarming, but also phenomena that resemble equilibrium phase behavior such as crystallization, condensation, and phase separation. As a consequence, there has been considerable interest in exploring the applicability of equilibrium statistical physics concepts, such as pressure, chemical potential, and surface tension, to describe non-equilibrium steady states resembling phase coexistence. ${ }^{1-29}$

Very recently, it was shown by experiments and simulations that steady states of a granular gas under vibration resemble phase coexistence of a dilute gas and a dense liquid phase that follows the lever rule, whereas the coexisting densities are well predicted by a Maxwell equal-area construction to the equation of state. ${ }^{10,11}$ Additionally, it was observed that these granular gases form patterns that resemble spinodal decomposition with a coarsening dynamics that proceeds via the same spatio-temporal scaling laws as in equilibrium molecular fluids. ${ }^{10,11}$ In the case of molecular fluids, the coarsening is driven by a reduction of the interfacial area and thereby a minimization of the interfacial energy. For granular gases, it was found that the coarsening dynamics can indeed be explained by the emergence of a positive non-equilibrium surface tension that is predominately determined by the anisotropy in the kinetic energy part of the stress tensor in contrast to the surface tension in molecular fluids, which is mainly determined by energetic interactions. ${ }^{12}$

Another model system that has recently received huge interest is a system of active Brownian particles suspended

\footnotetext{
a) Electronic mail: s.paliwal@uu.nl

b) S. Paliwal and V. Prymidis contributed equally to this work.

c) Electronic mail: m.dijkstra@uu.nl
}

in a solvent that incessantly converts energy from the local environment into directed motion and is thus inherently outof-equilibrium. The self-propulsion can be generated through a variety of mechanisms, for example, by hydrodynamic flows around a bacterium, ${ }^{30-32}$ self-diffusiophoresis, ${ }^{33}$ bubble propulsion, ${ }^{34,35}$ local demixing of a near critical solvent mixture, ${ }^{36,37}$ thermophoresis, ${ }^{38}$ Marangoni flows, ${ }^{39,40}$ and selfelectrophoresis ${ }^{41}$. In the simplest model of active Brownian particles, the particles perform directed motion with a constant self-propulsion speed, whereas the Brownian motion is described by stochastic translational forces as well as stochastic rotational forces that alter the direction of the persistent motion. In the case where these particles interact with purely repulsive interactions, dense clusters of particles in a dilute phase were observed at sufficiently high self-propulsion speeds in numerical simulations and in theory, a phenomenon termed as motility-induced phase separation (MIPS). ${ }^{13-26}$ Using large system sizes and an elongated simulation box, a stable phase separation between dense and dilute phases separated by planar interfaces was also achieved. ${ }^{27}$ Remarkably, the mechanical interfacial tension as determined by integrating the anisotropy of the pressure tensor in these simulations turns out to be negative. In the case of a negative surface tension in equilibrium fluids, the system can lower its free energy by creating more interfaces, and hence the phase separation is unstable. This intuitive interpretation of a negative surface tension is thus at odds with the observation of a stable motility-induced phase separation, thereby questioning the mechanical definition of surface tension and its equilibrium-like interpretation.

On the other hand, phase separation has also been observed in systems of self-propelled particles interacting with attractive interactions. ${ }^{19-21,42-45}$ Interestingly, a reentrant phase behavior was found in simulations of active colloidal particles interacting via Lennard-Jones (LJ) interactions. ${ }^{20,21}$ Phase-separated states were observed at low as well as high 
activities, and homogeneous states were found at intermediate activities. ${ }^{20,21}$ At high activity, the phase separation resembles the motility-induced phase separation as observed for active repulsive particles, whereas for low activity, the phase separation is caused by the attractive particle interactions and a kinetically arrested attractive gel phase is observed reminiscent of spinodal decomposition. ${ }^{20,21}$ However, it is yet unknown whether the coarsening dynamics of the spinodal structure of active Brownian Lennard-Jones particles bears any similarities with that of molecular fluids.

We thus conclude that many out-of-equilibrium steady states show a behavior reminiscent to that observed for equilibrium fluids such as condensation, crystallization, and phase separation. More surprisingly, also the kinetics of the phase separation displays striking similarities with the equilibrium counterparts. Vibrated granular systems exhibit spinodal decomposition with a coarsening dynamics that emerges from the presence of a non-equilibrium positive interfacial tension, whereas active repulsive particles show motility-induced phase separation with a negative surface tension implying that work is released by creating more interfaces while keeping the volume of the system fixed. ${ }^{27}$ In order to gain more insight into the concept of an interfacial tension in non-equilibrium systems, we investigate the interfacial tension and stiffness of a vapour-liquid interface of active Lennard-Jones systems. Many reasons justify this choice of the system. First of all, the bulk and interfacial behavior as well as the critical behavior of passive Lennard-Jones systems have been extensively studied over the past decades and we are provided with a wealth of information on the equilibrium passive counterpart of this model. ${ }^{46-50}$ Second, the computational efficiency of the model makes it very convenient and attractive for computer simulations. Furthermore, and perhaps more importantly, the system undergoes a vapour-liquid phase transition due to particle attractions for very low but also high activities of the particles, which correspond to both quasi-equilibrium and fairly out-of-equilibrium regimes. It is thus an ideal system to study systematically the effect of self-propulsion on the properties of the phase transition and of the interface as one can slowly switch on the activity of the system and drive the system further out-of-equilibrium, contrary to the case of motility-induced phase separation.

To this end, we study the stable vapour-liquid phase coexistence of isotropic self-propelled Brownian particles interacting with a truncated and shifted Lennard-Jones potential using Brownian dynamics simulations. Here, our investigation builds upon our previous work, in which we determined the vapour-liquid binodals as a function of rotational diffusion rate and self-propulsion speed of active Lennard-Jones particles. ${ }^{45}$ We use the overdamped Langevin equation to simulate the dynamics of the particles considering an implicit solvent. In order to stabilize direct coexistence, we employ an elongated simulation box, in which the planar interfaces align with the shortest dimensions of the box. We measure the normal and tangential components of the pressure tensor in the direction perpendicular to the interface by employing a local expression for the pressure tensor in active systems. ${ }^{5,51-53}$ The non-equilibrium interfacial tension is measured by integrating the difference of the normal and tangential components of this pressure tensor. ${ }^{54}$ We calculate the non-equilibrium surface tension for different combinations of self-propulsion speed and rotational diffusion rate and demonstrate that the trends of the surface tension can be fitted by simple power laws. In addition, we also apply capillary wave theory to understand the non-equilibrium relationship of interfacial tension and stiffness coefficient.

This paper is organized as follows. In Sec. II, we describe our model and the dynamics used in our numerical study. In Sec. III, we present our method which we used to measure the pressure tensor profiles and surface tension. We then discuss the density and pressure profiles in Secs. IV A and IV B, respectively. We determine the non-equilibrium interfacial tension in Sec. IV C and show that the surface tension as a function of strength of particle attractions is well fitted by a simple power law. Finally, we present our results on the interfacial stiffness as obtained from the application of the capillary wave theory and equipartition theorem in Sec. IV D and discuss its relation to the surface tension measured in Sec. IV C. We end with some conclusions in Sec. V.

\section{MODEL AND METHODS}

We consider a three-dimensional system consisting of self-propelled spherical particles suspended in a solvent. The particles interact via a truncated and shifted Lennard-Jones potential $U\left(r_{i j}\right)$ given by

$$
\begin{aligned}
U\left(r_{i j}\right) & =U_{L J}\left(r_{i j}\right)-U_{L J}\left(r_{c u t}\right) & & r \leq r_{c u t} \\
& =0 & & r>r_{c u t}
\end{aligned}
$$

with

$$
U_{L J}\left(r_{i j}\right)=4 \epsilon\left[\left(\frac{\sigma}{r_{i j}}\right)^{12}-\left(\frac{\sigma}{r_{i j}}\right)^{6}\right],
$$

where $r_{i j}=\left|\mathbf{r}_{j}-\mathbf{r}_{i}\right|$ is the center-of-mass distance, $\mathbf{r}_{i}$ the position of particle $i, \sigma$ is the particle length scale, and $\epsilon$ is the strength of the particle interaction. We set the cutoff radius $r_{c u t}=2.5 \sigma$ in all our simulations. In addition, we associate a three-dimensional unit vector $\mathbf{u}_{i}$ with particle $i$ that indicates the direction of the self-propelling force.

To describe the translational and rotational motion of the individual colloidal particles inside the solvent, we use the overdamped Langevin equations

$$
\begin{aligned}
& \frac{d \mathbf{r}_{i}}{d t}=-\frac{1}{\eta} \sum_{j \neq i} \frac{\partial U\left(r_{i j}\right)}{\partial \mathbf{r}_{i}}+v_{0} \mathbf{u}_{i}+\sqrt{2 D_{t}} \mathbf{\Lambda}_{i}^{t}, \\
& \frac{d \mathbf{u}_{i}}{d t}=\sqrt{2 D_{r}}\left(\mathbf{u}_{i} \times \Lambda_{i}^{r}\right)
\end{aligned}
$$

where $D_{t}$ is the translational diffusion coefficient given by the Stokes-Einstein relation $D_{t}=1 /\left(\beta_{s} \eta\right), \eta$ is the damping coefficient due to drag forces from the solvent, $\beta_{s}=1 / k_{B} T_{s}$ is the inverse temperature of the solvent bath with $k_{B}$ as the Boltzmann constant and $T_{s}$ as the bath temperature. $D_{r}$ is the rotational diffusion coefficient and $v_{0}$ denotes the self-propulsion speed. The vectors $\boldsymbol{\Lambda}_{i}^{t}$ and $\boldsymbol{\Lambda}_{i}^{r}$ are unit-variance Gaussian distributed random vectors with zero mean and variation,

$$
\begin{aligned}
\left\langle\boldsymbol{\Lambda}_{i}^{t, r}(t)\right\rangle & =0, \\
\left\langle\boldsymbol{\Lambda}_{i}^{t, r}(t) \boldsymbol{\Lambda}_{j}^{t, r}\left(t^{\prime}\right)\right\rangle & =\mathbb{I}_{3} \delta_{i j} \delta\left(t-t^{\prime}\right),
\end{aligned}
$$


where $\mathbb{I}_{3}$ is the $3 \times 3$ identity matrix. The angular brackets $\langle\ldots\rangle$ denote an average over different realizations of the noise. We also normalize the unit vector $\mathbf{u}_{i}$ of each particle $i$, after each iteration of Eq. (3) in order to prevent a drift with time.

We perform Brownian dynamics simulations in an elongated box with dimensions $L \times L \times 6 L$ for all cases except in Sec. IV D. The elongated shape of the simulation box stabilizes a phase coexistence with a planar interface between the two phases in the simulations. We apply periodic boundary conditions in all three directions and fix our $z$-coordinate axis along the longest edge of the box. The number of particles in our simulations is approximately $N=2500$, and the density of the system is kept fixed for all simulations at $\rho \sigma^{3}=0.1333$. We numerically integrate the equations of motion, Eqs. (2) and (3), using the Euler-Maruyama scheme. ${ }^{55}$ We set $\sigma, 1 / \beta_{s}$, and $\tau=\sigma^{2} / D_{t}$ as our units of length, energy, and time, respectively. We use a time step $d t=2 \times 10^{-5} \tau$ for the numeric integration of the equations of motion. In equilibrium, $\epsilon$ is inversely proportional to $k_{B} T_{s}$ and either parameter could be varied to control the temperature. Here we keep the temperature of the bath fixed by keeping $\beta_{s}$ constant and vary $\epsilon$ to mimic the change in the temperature of the colloidal particles. We employ the dimensionless temperature $T=k_{B} T_{s} / \epsilon$ following Ref. 45. In addition, we define the Péclet number as $\mathrm{Pe}=v_{0} / \sigma D_{r}$, which parameterizes the persistence length of the active motion. We investigate the interfacial properties of the system in the Péclet number range of $0-8$. To change the Péclet number, we either increase the self-propulsion speed at a fixed rate of the rotational diffusion coefficient $\left(D_{r} \tau=20\right)$ or decrease the rotational diffusion coefficient at fixed selfpropulsion speed $\left(v_{0} \tau \sigma^{-1}=8\right)$. The choice of parameters studied here is exactly the same as in Ref. 45, where the vaporliquid binodals have been mapped out. Note that in Ref. 44, it was shown that a percolating network state could separate the fluid from the vapour-liquid coexistence region when the system was sufficiently far from equilibrium. However, for the parameters studied here, as argued in Ref. 45, there are no signatures of a percolating state within the coexistence regions.

For each set of simulation parameters, we let the systems reach a steady state by running the simulations for $\approx 600 \tau$ and then collect data for another $1200 \tau$ by measuring the quantities of interest every 100 time steps. We also fix the center of mass of the system at the origin of the $z$-axis in order to prevent the drift of the liquid slab that coexists with the gas by regularly shifting the coordinates of the particles at fixed time intervals.

\section{PRESSURE TENSOR}

The concept of a non-equilibrium pressure in active systems has received a lot of attention in recent years. Various approaches have been followed to derive a microscopic expression for the bulk pressure of an active-particle system. It has already been shown that an extra swim pressure contribution arises due to the self-propulsion of the particles in active matter using a micromechanical, ${ }^{25}$ a virial,,${ }^{7,9,18,51}$ or a stochastic thermodynamics formulation. ${ }^{9}$ Solon and co-workers have argued that in the case of an active system interacting with anisotropic interactions, the pressure depends on the wallparticle interactions, which implies that pressure is not a state function. ${ }^{52}$ In the case of isotropic interactions, the various approaches yield consistent results for the microscopic expression of the bulk pressure. Furthermore, a microscopic definition for the local stress tensor has been derived from the stationary probability distribution function by using the Fokker-Planck equation. ${ }^{4-6,27,52,53,56,57}$

In order to simulate direct coexistence between active vapour and liquid phases, we employ an elongated simulation box with the long axis along the $z$-direction and in which the two coexisting phases are separated by interfaces parallel to the $x y$-plane. Hence, the system is only inhomogeneous in the $z$-direction, and consequently, the pressure tensor contains only two independent components: the normal component along the direction perpendicular to the interfaces, $P_{N}(z)=P_{z z}(z)$, and the transverse component, $P_{T}(z)=\left(P_{x x}(z)\right.$ $\left.+P_{y y}(z)\right) / 2$, which is the average of the $x x$ and $y y$ components due to the symmetry of the system in the $x y$-plane. The non-diagonal components of the pressure tensor vanish due to hydrostatic equilibrium, which we verified in our simulations.

As described in Refs. 5, 53, and 57, the microscopic local pressure tensor for interacting spherical particles without torques is derived using the steady state probability distribution function $\psi(\mathbf{r}, \mathbf{u})=\left\langle\sum_{i=1}^{N} \delta\left(\mathbf{r}-\mathbf{r}_{i}\right) \delta\left(\mathbf{u}-\mathbf{u}_{i}\right)\right\rangle$, where $\mathbf{r}$ $=(x, y, z)$ is the 3 -dimensional spatial coordinate and unit vector $\mathbf{u}$ is the analog for orientation. The diagonal spatial components of this local pressure tensor, $P_{\alpha \alpha}(z)$, consist of an ideal gas contribution, a virial contribution, and a swim pressure contribution,

$$
P_{\alpha \alpha}(z)=P_{\alpha \alpha}^{\mathrm{id}}(z)+P_{\alpha \alpha}^{\mathrm{vir}}(z)+P_{\alpha \alpha}^{\mathrm{swim}}(z)
$$

The ideal component of the pressure reads as

$$
P_{\alpha \alpha}^{\mathrm{id}}(z)=\rho(z) k_{B} T_{s}
$$

with $\rho(z)$ as the density profile, and the virial and swim contributions due to the particle interactions and self-propulsion are given by

$$
P_{\alpha \alpha}^{\mathrm{vir}, \operatorname{swim}}(z)=\frac{1}{L^{2}} \int d x \int d y P_{\alpha \alpha}^{\mathrm{vir}, \operatorname{swim}}(\mathbf{r})
$$

with

$$
P_{\alpha \alpha}^{\mathrm{vir}}(\mathbf{r})=-\int_{\mathbf{r}_{\alpha}^{\prime \prime}<\mathbf{r}_{\alpha}} d \mathbf{r}_{\alpha}^{\prime \prime} \int d \mathbf{r}^{\prime} \rho^{(2)}\left(\mathbf{r}^{\prime \prime}, \mathbf{r}^{\prime}\right) \frac{\partial U\left(\left|\mathbf{r}^{\prime \prime}-\mathbf{r}^{\prime}\right|\right)}{\partial \mathbf{r}_{\alpha}^{\prime \prime}},
$$

where $\rho^{(2)}\left(\mathbf{r}, \mathbf{r}^{\prime}\right)=\int d \mathbf{u} \int d \mathbf{u}^{\prime} \psi^{(2)}\left(\mathbf{r}, \mathbf{u}, \mathbf{r}^{\prime}, \mathbf{u}^{\prime}\right)$ is the spatial two-body correlation function with the full two-body correlation function $\psi^{(2)}\left(\mathbf{r}, \mathbf{u}, \mathbf{r}^{\prime}, \mathbf{u}^{\prime}\right) \equiv\left\langle\sum_{i=1}^{N} \sum_{j \neq i}^{N} \delta\left(\mathbf{r}-\mathbf{r}_{i}\right) \delta(\mathbf{u}\right.$ $\left.\left.-\mathbf{u}_{i}\right) \delta\left(\mathbf{r}^{\prime}-\mathbf{r}_{j}\right) \delta\left(\mathbf{u}^{\prime}-\mathbf{u}_{j}\right)\right\rangle$. Here, the angular brackets $\langle\ldots\rangle$ denote a time average over steady states. The integral is over the $\alpha$ component of the vector $\mathbf{r}^{\prime \prime}$ and we define $\mathbf{r}_{\beta}^{\prime \prime}=\mathbf{r}_{\alpha}, \forall \beta$ $\neq \alpha$. 
The local swim pressure contribution is given by

$$
\begin{aligned}
P_{\alpha \alpha}^{\mathrm{swim}} & (\mathbf{r}) \\
= & \frac{k_{B} T_{s} v_{0}^{2}}{2 D_{t} D_{r}} \int d \mathbf{u} \psi(\mathbf{r}, \mathbf{u}) \mathbf{u}_{\alpha} \mathbf{u}_{\alpha} \\
& -\frac{v_{0}}{2 D_{r}} \int d \mathbf{u} \int d \mathbf{r}^{\prime} \int d \mathbf{u}^{\prime} \psi^{(2)}\left(\mathbf{r}, \mathbf{u}, \mathbf{r}^{\prime}, \mathbf{u}^{\prime}\right) \frac{\partial U\left(\left|\mathbf{r}-\mathbf{r}^{\prime}\right|\right)}{\partial \mathbf{r}_{\alpha}} \mathbf{u}_{\alpha} \\
& -\frac{k_{B} T_{s} v_{0}}{2 D_{r}} \frac{\partial}{\partial \mathbf{r}_{\alpha}} \int d \mathbf{u} \psi(\mathbf{r}, \mathbf{u}) \mathbf{u}_{\alpha} .
\end{aligned}
$$

In our simulations, we measure the density profiles $\rho(z)$ and the normal and transverse components of the pressure tensor profiles, $P_{N}(z)$ and $P_{T}(z)$, by dividing the system into small slabs of width $\Delta z=0.1 \sigma$ and area $L^{2}$ and by measuring the local average quantities in each bin. The local density $\rho\left(z_{k}\right)$ in bin $k$ centered around $z=z_{k}$ is measured using

$$
\rho\left(z_{k}\right)=\frac{\left\langle n\left(z_{k}\right)\right\rangle}{\Delta V},
$$

with $\left\langle n\left(z_{k}\right)\right\rangle$ as the average number of particles in bin $k$ and $\Delta V=L^{2} \Delta z$ as the volume of a bin. Using Ref. 58, we rewrite and measure the virial contribution in bin $k$ as follows:

$$
P_{\alpha \alpha}^{\operatorname{vir}}\left(z_{k}\right)=\frac{1}{2 \Delta V}\left\langle\sum_{i=1}^{n\left(z_{k}\right)} \sum_{j \neq i}^{N} \frac{\mathbf{r}_{i j, \alpha}}{r_{i j}} \frac{d U\left(r_{i j}\right)}{d r_{i j}} \int_{C_{i j} \in \Delta z_{k}} d \mathbf{l}_{\mathbf{L}}\right\rangle,
$$

with $r_{i j}=\left|\mathrm{r}_{i j}\right|=\left|\mathrm{r}_{j}-\mathrm{r}_{i}\right|$ as the center-of-mass distance of particles $i$ and $j$. The variable of integration $\mathbf{l}_{\alpha}$ is along the $\alpha$ component of the integration contour $C_{i j}$ from $\mathrm{r}_{i}$ to $\mathrm{r}_{j}$. The integral denotes that the virial contribution to the pressure of particle pair $i$ and $j$ is due to the part of $C_{i j}$ that lies inside the respective bin within the coarse-grained Irving-Kirkwood approximation. ${ }^{58}$

Finally, the local swim pressure can be measured in each bin $k$ using

$$
\begin{aligned}
P_{\alpha \alpha}^{\mathrm{swim}}\left(z_{k}\right)= & \frac{k_{B} T_{s} v_{0}^{2}}{2 D_{t} D_{r} \Delta V}\left\langle\sum_{i=1}^{n\left(z_{k}\right)} \mathbf{u}_{i, \alpha}^{2}\right\rangle \\
& -\frac{v_{0}}{2 D_{r} \Delta V}\left\langle\sum_{i=1}^{n\left(z_{k}\right)} \sum_{j \neq i}^{N} \frac{\mathbf{r}_{i j, \alpha}}{r_{i j}} \frac{d U\left(r_{i j}\right)}{d r_{i j}} \mathbf{u}_{i, \alpha}\right\rangle \\
& -\frac{k_{B} T_{s} v_{0}}{2 D_{r} \Delta V} \frac{\partial}{\partial \mathbf{r}_{i j, \alpha}}\left\langle\sum_{i=1}^{n\left(z_{k}\right)} \mathbf{u}_{i, \alpha}\right\rangle .
\end{aligned}
$$

Note that the last term in Eq. (13) is a term not present in the case of an isotropic bulk phase as discussed previously in Refs. 5, 6, and 51. This term is non-zero for systems with finite polarization, defined as $\mathbf{m}\left(z_{k}\right)=\left\langle\sum_{i=1}^{n\left(z_{k}\right)} \mathbf{u}_{i}\right\rangle / \Delta V$, for instance, at interfaces or surfaces, but disappears in the homogeneous bulk phase of the fluid.

\section{RESULTS}

Using Brownian dynamics simulations, we investigate the interfacial properties of vapour-liquid interfaces in systems of active Lennard-Jones particles for different combinations of self-propulsion speed and rotational diffusion rate, i.e., for varying Péclet numbers.

\section{A. Density and orientation profiles}

To start our investigation, we first measure and plot the average density profile $\rho(z)$ to verify coexistence of vapour and liquid phases in our simulation box. We choose the selfpropulsion speed, density, and temperature such that they correspond to a state point that lies well-inside the two-phase coexistence region as determined in Ref. 45. A typical configuration of a steady state exhibiting vapour-liquid phase coexistence of $N=2500$ active Lennard-Jones particles is shown in Fig. 1(a), and the corresponding density profile is presented in Fig. 1(b). We find that the density profiles are similar to passive equilibrium profiles and can be well fitted to a hyperbolic tangent function

$$
\rho(z)=\frac{1}{2}\left(\rho_{l}+\rho_{v}\right)-\frac{1}{2}\left(\rho_{l}-\rho_{v}\right) \tanh \left[\frac{2\left(z-z_{0}\right)}{D}\right],
$$

where $\rho_{l}$ and $\rho_{v}$ are the corresponding bulk liquid and vapour coexisting densities, $z_{0}$ is the location of the plane satisfying an equal-area construction, and $D$ represents the thickness of the interface. We fit the above equation to the right and left halves of the box $(z>0$ and $z<0)$ separately using $z_{0}$ and $D$ as fitting parameters and obtain the bulk densities $\rho_{l}$ and $\rho_{v}$ from the mean of the two fits. We denote the interfacial regions of width $D$ as shaded grey areas in Figs. 1 and 2 .

In Fig. 1(c) we plot the local average orientation of particles $\mathbf{u}(z)=\langle\mathbf{m}(z)\rangle /\langle\rho(z)\rangle$. It is evident that the particles tend to orient themselves along the normal direction at the interfaces, and the peak of the orientation profile does not coincide with the estimated position of the interface $z_{0}$ (dotted lines). On average, the particles tend to orient themselves with the direction of self-propulsion towards the less-dense (vapour) phase. This asymmetry in the average orientation is easily explained

(a)

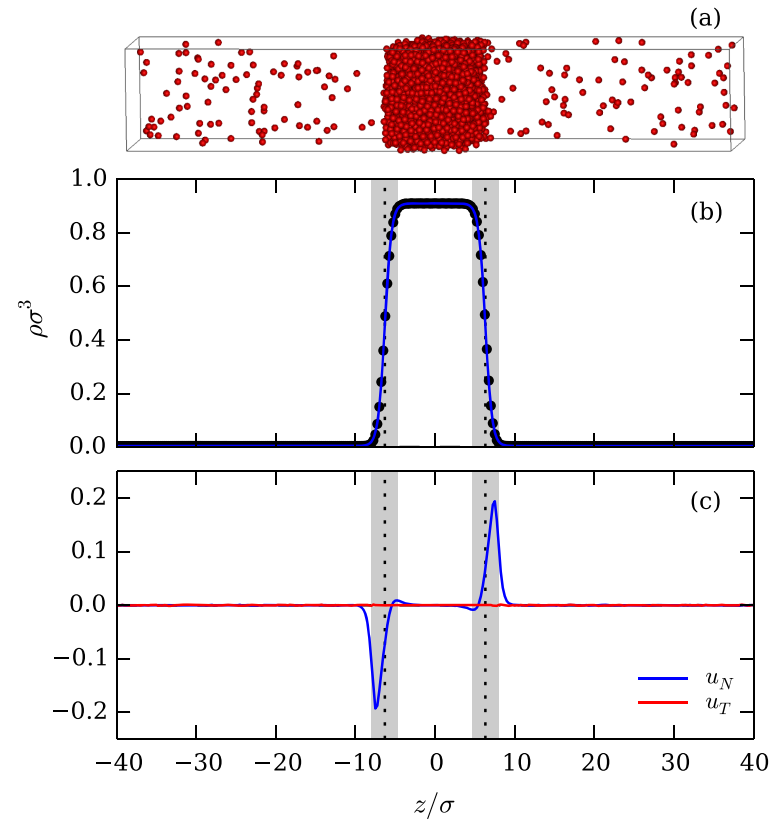

FIG. 1. (a) A typical snapshot of the simulation box showing a steady state of a vapour-liquid phase coexistence of active Lennard-Jones particles with a selfpropulsion speed $v_{0} \tau \sigma^{-1}=28$ at a temperature $k_{B} T_{s} / \epsilon=0.2$ and rotational diffusion rate $D_{r} \tau=20$. The dense liquid slab is in the middle of the box and is separated from the vapour phase by two planar interfaces. (b) Local density profile $\rho(z)$. Data points correspond to simulation measurements and the continuous line is the fit using Eq. (14). Dotted lines indicate the location $z_{0}$ of the interface according to Eq. (14) and the shaded areas denote the interfacial regions $\left(z_{0}-D / 2, z_{0}+D / 2\right)$. (c) Profiles of the components of the orientation vector $\mathbf{u}(z)=\langle\mathbf{m}(z)\rangle /\langle\rho(z)\rangle\left[u_{N}=u_{z}\right.$ and $\left.u_{T}=\left(u_{x}+u_{y}\right) / 2\right]$. 


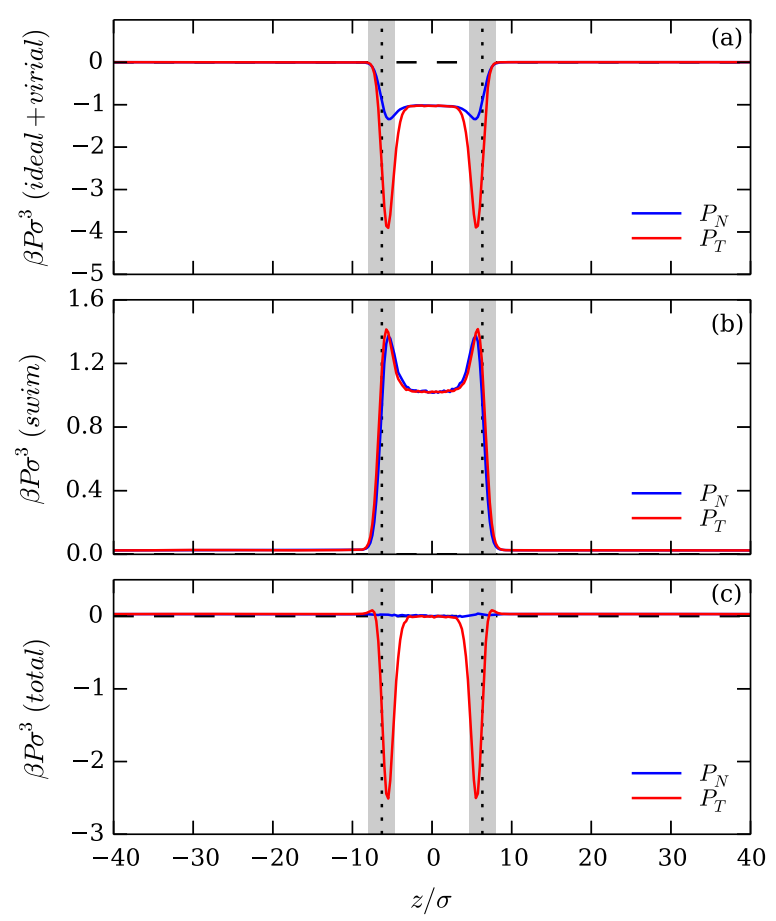

FIG. 2. Normal and tangential components of (a) the ideal and virial pressure tensors $P_{N, T}^{\mathrm{id}}(z)+P_{N, T}^{\mathrm{vir}}(z)$, (b) the swim pressure tensor $P_{N, T}^{\mathrm{swim}}(z)$, and (c) the total pressure tensor $P_{N, T}^{\text {tot }}(z)=P_{N, T}^{\text {id }}(z)+P_{N, T}^{\mathrm{vir}}(z)+P_{N, T}^{\mathrm{swim}}(z)$. The normal component of $P_{N}^{\text {tot }}(z)$ is constant for all $z$ indicating mechanical equilibrium. The tangential component shows distinctive peaks at the two interfaces. The simulation parameters are the same as in Fig. 1.

by assuming a zero net velocity at the interface: particles at the interface that point towards the dense phase have a larger average velocity than particles that point towards the dilute phase due to the net attractive force towards the liquid. Thus, more particles need to point outwards in order to balance the asymmetry in velocities. It is also important to note that this preferential ordering is only along the normal $(z)$ direction. There is no net orientation along the tangential plane $(x y)$ as the system is isotropic in this plane. We note that in the case of MIPS, where the activity drives the phase separation, the orientations tend to be exactly reverse, with the preferred orientation of particles at the interfaces being towards the denser phase.

We also find that at fixed activity, which for our system translates to fixed self-propulsion speed and rotational diffusion coefficient, the shape of the orientation profile along the interface as well as the interfacial width $D$ becomes broader upon increasing $T$ or equivalently upon decreasing the strength of attraction between particles. The broadening of the interface as the system moves towards its "critical point" is completely analogous to what is observed in the passive LJ system. ${ }^{47}$ Also, at fixed temperature $T$, the interfacial region becomes broader as the activity increases. This observation is compatible with Ref. 45, which showed that higher attraction strength is needed to induce phase separation upon increasing activity and is also consistent with other older studies. ${ }^{19,42,43}$

\section{B. Pressure profiles}

Subsequently, we measure the different contributions to the normal and tangential components of the pressure tensor using Eqs. (11)-(13) for our phase-separated systems. Figure 2 shows typical profiles of the normal and tangential components of the ideal and virial pressure tensors, the swim pressure tensor, and the total pressure tensor. Below we discuss the various contributions as well as the total pressure profiles separately.

In passive systems, mechanical equilibrium requires a constant normal component of the total pressure, which simply consists of an ideal and a virial contribution, in the direction perpendicular to the interfaces. However, a net imbalance of the interaction forces along the tangential plane causes the tangential component of the pressure to be smaller on average than the normal component along the interfacial region. This inequality of the pressure components at the interface leads to a positive surface tension in the case of equilibrium fluids. ${ }^{59,60}$ In the case of our active LJ system, Fig. 2(a) shows that the normal component of the sum of the ideal and the virial pressures is not constant across the system and that the liquid has a smaller bulk pressure than the vapour phase. Thus, mechanical equilibrium is not established simply by considering the virial and the ideal components of the pressure. Moreover, the tangential component is also not equal at the bulk of the two coexisting phases though it is reassuringly equal to the normal component in the bulk. It is also smaller on average than the normal component of the pressure along the interface, similar to the passive case. Note that the behavior of the sum of the ideal and the virial components of the pressure is reversed with respect to their respective profiles in the case of MIPS. ${ }^{27}$ In that case, the ideal and virial components are higher in the dense phase than in the dilute phase.

The swim pressure, as we see in Fig. 2(b), is also not equal in the two phases for both the normal and tangential components. Its magnitude is larger in the liquid phase than the vapour phase where it is essentially zero. Also, both components show peaks along the interfaces. Again, the pressure profile has an opposite behavior with respect to the case of MIPS, where the swim pressure is higher in the dilute phase than in the dense phase. ${ }^{27}$

In Fig. 2(c) we show the total pressure, that is, the sum of the ideal, the virial, and the swim pressures. Reassuringly, the normal component now becomes constant throughout the system, as is required for mechanical equilibrium. We wish to emphasize here that the gradient term of the form $\partial_{\alpha} \mathbf{m}_{\alpha}$ in the swim pressure, Eq. (13), needs to be included in the total pressure to obtain a perfectly flat profile for the normal component of the pressure tensor at the interface. This term is obviously zero in the bulk of the system but its magnitude along the interface increases as the activity of the system is increased. The tangential component of the total pressure is also equal in the two bulks but has negative peaks at the interfaces. This is again similar to the case of equilibrium systems and leads to a positive surface tension, as we will discuss in more detail in Sec. IV C. In the case of MIPS, the total pressure profiles again recover to equal pressures in the bulks upon including the swim pressure but the tangential component has a different behavior at the interface than the ones shown in Fig. 2(c). ${ }^{27}$ The tangential component in that case has positive peaks which translate into a negative vapour-liquid interfacial tension. 


\section{Surface tension}

In the case of equilibrium fluids, the surface tension $\gamma$ of an interface that separates two coexisting bulk phases can be defined in various ways. ${ }^{61}$ The surface tension can be defined thermodynamically as the difference in grand potential between a phase-separated system with an interface and a homogeneous bulk system, which are both at the same coexisting bulk chemical potential, divided by the surface area of the interface. Using this definition, the vapour-liquid interfacial tension can be determined in simulations by measuring the grand canonical probability distribution function of observing $N$ particles in a volume $V$ at a fixed chemical potential $\mu$ and temperature $T$. This probability distribution function can be measured very accurately using successive umbrella sampling in grand canonical Monte Carlo simulations. ${ }^{62}$ Using the histogram reweighting technique, one can then determine the chemical potential corresponding to bulk coexistence using the equal-area rule for the vapour and liquid peaks. ${ }^{61,62}$ The interfacial tension can be determined from the difference in the maximum of the peaks and the minimum. ${ }^{63-65}$ Alternatively, one can also determine the surface tension by measuring the width of the interface, which is determined by an intrinsic width and a broadening due to capillary wave fluctuations. Using the equipartition theorem, one can relate the meansquare fluctuations due to capillary waves to the interfacial tension, and hence the interfacial tension can be determined by measuring the capillary wave broadening. ${ }^{61,66-69}$ It is important to note that the method to determine the interfacial tension from the probability distribution is based on grand canonical Monte Carlo simulations and relies on a knowledge of the statistical weight corresponding to the grand canonical ensemble. The second method employs the equipartition theorem, which is derived by assuming a Boltzmann distribution. Finally, the interfacial tension can be defined as the mechanical work required to enlarge the interface. Using the condition of hydrostatic equilibrium, the surface tension can be defined as the integral of the difference of the two pressure tensor components,

$$
\gamma=\frac{1}{2} \int_{-L / 2}^{L / 2}\left[P_{N}(z)-P_{T}(z)\right] d z,
$$

where we assume that the system is only inhomogenous in the $z$-direction with the two planar interfaces parallel to the $x y$-plane. The factor $\frac{1}{2}$ comes from the presence of two interfaces in a simulation with periodic boundary conditions. For equilibrium fluids, all these definitions for the interfacial tension coincide. In the case of non-equilibrium systems such as the active LJ system, the statistical weights of the different ensembles are unknown, which precludes the use of Monte Carlo simulations for determining the interfacial tension from a probability distribution function. We therefore resort to the mechanical definition of the surface tension by employing Eq. (15). In addition, we measure the interfacial width in Brownian dynamics simulations and naively assume the equipartition theorem to hold even though it is based on a statistical ensemble average. We present and discuss our results below using the mechanical definition and later in Sec. IV D for the application of capillary wave theory.
Following Ref. 27, we determine the surface tension using the mechanical route [Eq. (15)], where we also include the contribution from the swim pressure in the total pressure in order to satisfy the hydrostatic equilibrium condition. Note that the gradient term of the form $\partial_{\alpha} \mathbf{m}_{\alpha}$ in the swim pressure [Eq. (13)], which is essential in order to obtain a flat profile of the normal pressure component across the interface, does not contribute to the surface tension. Using Eq. (15) and the total pressure profiles as exemplarily shown in Fig. 2(c), we determine the surface tension $\gamma$ for a wide range of parameters of the active system following two paths that drive the system out of equilibrium. To this end, we either increase the self-propulsion speed at a fixed rotational diffusion rate $\left(D_{r} \tau=20\right)$ or decrease the rotational diffusion coefficient at a fixed self-propulsion speed $\left(v_{0} \tau \sigma^{-1}=8\right)$. For the first path, we choose a high value for the rotational diffusion coefficient in order to minimize the regime of percolating states in the state diagram. ${ }^{44}$ Note that the equilibrium limits of these two paths are not equivalent as the $D_{r} \rightarrow \infty$ limit does not coincide with the $v_{0} \rightarrow 0$ limit. The second limit corresponds to the equilibrium LJ system with temperature $k_{B} T_{s} / \epsilon$ while the first limit corresponds to a passive system with a higher effective temperature. The systems we examine have a Péclet number in the range $0-8$, where the Péclet number is defined as $\mathrm{P} e=v_{0} / D_{r} \sigma$, so that we probe the equilibrium limit as well as systems where self-propulsion plays a much more important role in the dynamics than translational diffusion. However, in all cases, we are well below the onset of MIPS, ${ }^{14}$ i.e., $\mathrm{Pe} \sim 50$.

We plot the surface tension $\gamma \sigma^{2} / \epsilon$ as a function of temperature $T=k_{B} T_{s} / \epsilon$ in Figs. 3(a) and 4(a) for constant rotational diffusion coefficient and constant speed of self-propulsion, respectively. Note that we always measure a positive surface tension, contrary to the case of MIPS, ${ }^{27}$ and the magnitude of the surface tension is in the same range $\left(\gamma \sigma^{2} / \epsilon \sim 1\right)$ as in the equilibrium system. ${ }^{47} \mathrm{We}$ also find that the surface tension decreases upon increasing the temperature towards the critical temperature $T_{c}$ as the density difference between the coexisting phases decreases, which is similar to equilibrium systems for which the surface tension vanishes at the critical point.

Next, we examine the scaling of the surface tension $\gamma$ with temperature $T$ as the system departs from the equilibrium regime by increasing the activity of the Lennard-Jones particles. In the case of equilibrium systems, $\gamma$ scales with temperature as

$$
\gamma \sigma^{2} / \epsilon=A\left(1-T / T_{c}\right)^{c},
$$

where $A$ denotes a dimensionless constant, $T_{c}$ denotes the critical temperature, and $c$ denotes a critical exponent. In the case of equilibrium systems, $c=2 v$ with $v=0.63$ as the critical exponent of the bulk correlation length of the system. ${ }^{48}$ Here, we examine whether the surface tension for our active system follows a scaling with temperature similar to Eq. (16) and treat $A, T_{c}$, and the exponent $c$ as fit parameters.

In Figs. 3(b) and 4(b), we plot the resulting fits which are offset for clarity. The same fits are shown as solid lines in Figs. 3(a) and 4(a). We find that they fit well to the measured data in the examined parameter space. We thus observe that the scaling of the surface tension with temperature can be 

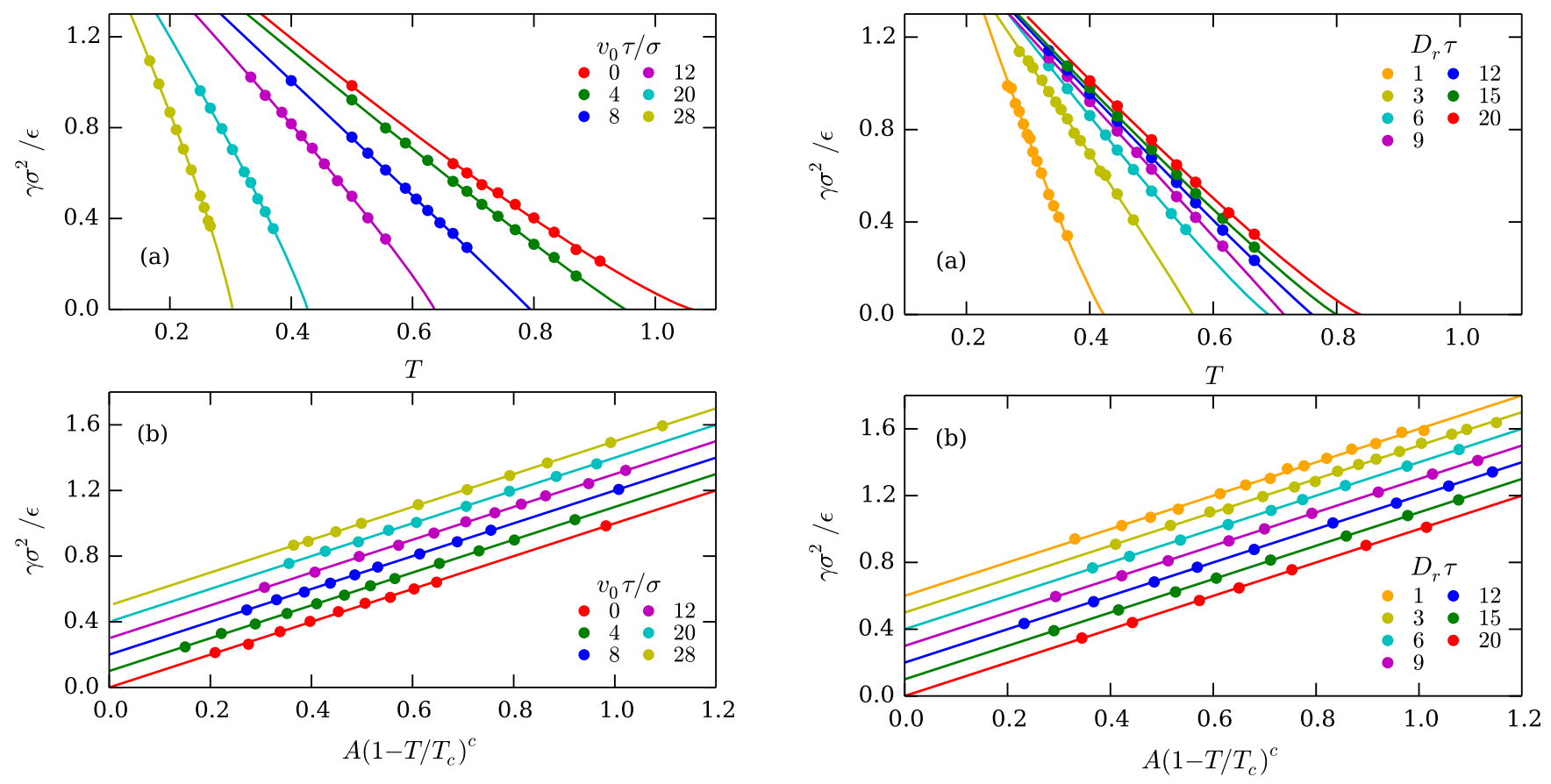
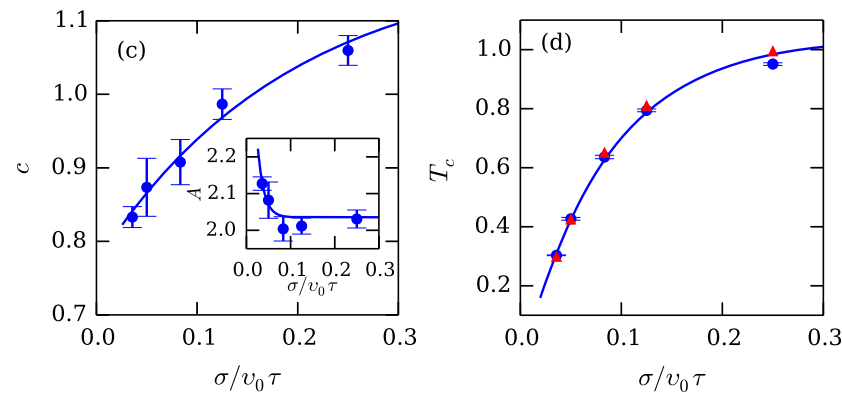

FIG. 3. Vapour-liquid interfacial tension $\gamma \sigma^{2} / \epsilon$ as a function of (a) temperature $T=k_{B} T_{s} / \epsilon$ and (b) scaled temperature, for an active Lennard-Jones system (circles) with a rotational diffusion rate $D_{r} \tau=20$ for varying selfpropulsion speeds $v_{0} \tau / \sigma$ as obtained from Eq. (15) and corresponding fits using Eq. (16). Results in (b) are offset for clarity. (c) Fit parameters $c$ and $A$ (inset) as a function of the inverse self-propulsion speed $\sigma /\left(v_{0} \tau\right)$ with errorbars in the estimate of these parameters and the corresponding fit using Eq. (17). (d) The scaling of the estimated $T_{c}$ as obtained from the scaling of the surface tension $\gamma$ (circles) and the values obtained from the scaling of the coexistence densities from Ref. 45 (triangles) along with the corresponding fits using Eq. (17).

captured by Eq. (16) even for the active systems considered here. Note that these fits also give us an estimate for the critical temperature of the system in the limit $\gamma=0$ for different degrees of activity.

We now examine the scaling of the fit parameters $A, c$, and $T_{c}$ upon increasing the activity. The results for the parameters $A$ and $c$ are plotted in Figs. 3(c) and 4(c). We find that driving the system further away from equilibrium by increasing the self-propulsion speed at a fixed rotational diffusion coefficient, the value of the exponent $c$ decreases, while the parameter $A$ increases [Fig. 3(c)]. The exponent $c$ moves away from its equilibrium value $c=1.21-1.26^{47,70}$ to values less than unity. We find a similar scaling in the case where the system is driven out of equilibrium by decreasing the rotational diffusion coefficient at a fixed self-propulsion speed, i.e., $c$ decreases, while $A$ increases. However, the exponent $c$ appears to increase again for very low values of the rotational diffusion coefficient
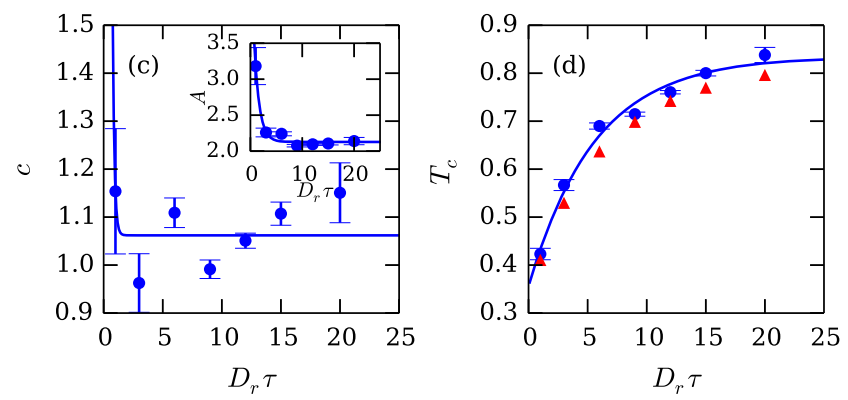

FIG. 4. Vapour-liquid interfacial tension $\gamma \sigma^{2} / \epsilon$ as a function of (a) temperature $T=k_{B} T_{s} / \epsilon$ and (b) scaled temperature, for an active Lennard-Jones system (circles) with a self-propulsion speed $v_{0} \tau \sigma^{-1}=8$ and varying rotational diffusion rates $D_{r} \tau$ as obtained from Eq. (15) and corresponding fits using Eq. (16). Results in (b) are offset for clarity. (c) Fit parameters $c$ and $A$ (inset) as a function of the rotational diffusion rates $D_{r} \tau$ with errorbars and the corresponding fit using Eq. (18). (d) The scaling of the estimated $T_{c}$ as obtained from the scaling of the surface tension $\gamma$ (circles) and the values obtained from the scaling of the coexistence densities from Ref. 45 (triangles) along with the corresponding fits using Eq. (18).

as shown in Fig. 4(c). Unfortunately, large errorbars in the fits for this regime prevent us from making any definite conclusions on the dependence of the exponent $c$ on the activity of the system for high Péclet numbers.

Furthermore, the scaling of the critical temperature with the self-propulsion force is in accordance with the findings of Ref. 45, showing that $T_{c}$ decreases upon increasing activity. In Figs. 3(d) and 4(d), we plot the cases of varying self-propulsion speed and varying rotational diffusion coefficient respectively, both demonstrating the trend. Lastly, we also compare the critical temperature as determined from the scaling of the order parameter $\Delta \rho=\rho_{l}-\rho_{v}$ from Ref. 45 in Figs. 3(d) and 4(d). We find that the two values of the critical temperature as evaluated from the two different routes, i.e., via the scaling of the order parameter and via the scaling of the surface tension with temperature, are very close to each other in the case of varying self-propulsion speed but the agreement is not as 
good in the case of varying rotational diffusion at a constant self-propulsion speed. Nonetheless, the values from the two routes are still close to one another and follow a similar scaling.

Additionally, we show empirical fits for the dependence of the parameters $A, c$, and $T_{c}$ on the self-propulsion speed $v_{0}$ and the rotational diffusion coefficient $D_{r}$, respectively. All three parameters are fitted by simple exponential scalings, namely,

$$
\begin{aligned}
A\left(v_{0}\right), c\left(v_{0}\right), T_{c}\left(v_{0}\right) & =a_{1} e^{-a_{2} \sigma / v_{0} \tau}+a_{3}, \\
A\left(D_{r}\right), c\left(D_{r}\right), T_{c}\left(D_{r}\right) & =b_{1} e^{-b_{2} D_{r} \tau}+b_{3},
\end{aligned}
$$

where $a_{1}, a_{2}, a_{3}$ and $b_{1}, b_{2}, b_{3}$ are again fit parameters. These fits capture the scaling of the exponent $c$ and the parameter $A$ [Figs. 3(c) and 4(c)] for varying self-propulsion speeds $v_{0}$ [Eq. (17)] and rotational diffusion coefficients $D_{r}$ [Eq. (18)]. The fit for $c$ obviously fails for varying rotational diffusion coefficients [Fig. 4(c)], but we still present it for consistency. The scaling of the critical temperature $T_{c}$ is shown in Figs. 3(d) and 4(d) along with the values from Ref. 45. The fit parameters $a_{1}, a_{2}, a_{3}$ and $b_{1}, b_{2}, b_{3}$ providing the scaling of $A$ and $c$ as well as the two values for $T_{c}$ are listed in Tables I and II for varying self-propulsion speeds and rotational diffusion coefficients, respectively.

Before closing this section, it is important to remark that we use Eq. (16) merely as a fit to our results and that we do not identify the associated fit parameters with the critical point and critical exponents of the current system. In fact, we have not yet demonstrated the existence of a critical point for active LJ systems as we are unable to obtain reliable data on the vapourliquid phase coexistence in the critical regime due to the small system sizes that we used in our simulations. Nonetheless, it is instructive to compare the equilibrium limit of our measurements to their known equilibrium values, with the equilibrium limit corresponding to the limits $v_{0} \tau / \sigma \rightarrow 0$ and $D_{r} \tau \rightarrow \infty$ for the results presented in Tables I and II, respectively. We find that our estimates for the critical point and exponents are rough, yet reasonable. Specifically, we estimate the equilibrium critical point at $T_{c}=1.041$, while recent finite size scaling studies report $T_{c}=1.187 .{ }^{71}$ Furthermore, we find the exponent $c=1.181$ and 1.062 , while the literature reads as $c$ $=1.21-1.26^{47,70}$

\section{Interface fluctuations and stiffness}

In this section, we study the scaling of the interfacial width as a function of the area of the interface, which allows us to measure the stiffness of the interface. Subsequently we attempt to connect the estimated value for the stiffness to the value of the surface tension obtained in Sec. IV C.

TABLE I. Fitting parameters of Eq. (17) for an active Lennard-Jones system with a rotational diffusion rate $D_{r} \tau=20$ and varying self-propulsion speeds $v_{0} \tau \sigma^{-1}$.

\begin{tabular}{lcccc}
\hline \hline & $A$ & $c$ & \multicolumn{1}{c}{$T_{c}$} & $T_{c}{ }^{\mathrm{a}}$ \\
\hline$a_{1}$ & 1.159 & -0.410 & -1.113 & -0.184 \\
$a_{2}$ & 70.01 & 5.257 & 11.810 & 12.33 \\
$a_{3}$ & 2.035 & 1.181 & 1.041 & 1.066 \\
\hline \hline
\end{tabular}

${ }^{a}$ Values from Ref. 45 .
TABLE II. Fitting parameters of Eq. (18) for an active Lennard-Jones system with a self-propulsion speed $v_{0} \tau \sigma^{-1}=8$ and varying rotational diffusion rates $D_{r} \tau$.

\begin{tabular}{rrrrr}
\hline \hline & $A$ & $c$ & $T_{c}$ & \multicolumn{1}{c}{$T_{c}{ }^{\mathrm{a}}$} \\
\hline$b_{1}$ & 2.840 & 109.99 & -0.478 & -0.470 \\
$b_{2}$ & 0.993 & 7.095 & 0.178 & 0.156 \\
$b_{3}$ & 2.128 & 1.062 & 0.834 & 0.818 \\
\hline \hline
\end{tabular}

${ }^{a}$ Values from Ref. 45.

For equilibrium systems, capillary wave theory provides a connection between the fluctuations of an interface and its stiffness coefficient or interfacial tension. ${ }^{66-69}$ Capillary wave theory ${ }^{72}$ describes the broadening of an intrinsic interface of width $w_{0}$ due to thermal fluctuations. The capillary wave broadening depends primarily on the interfacial tension and the area of the interface and can be calculated by using the equipartition theorem and summing over the mean-square fluctuations of all allowed excitation modes of the interface. We refer the reader to Refs. 66 and 67 for more details and present here only the result. According to capillary wave theory, ${ }^{66,67}$ the total interfacial width $w$ can be written as the sum of an intrinsic part $w_{0}$ and a contribution due to capillary wave fluctuations,

$$
w^{2}=w_{0}^{2}+\frac{1}{\kappa} \ln \left(\frac{L}{\xi}\right),
$$

where $\xi$ is the bulk correlation length and $\kappa$ is the stiffness coefficient, which parametrizes the energy penalty for deformations of the interface with dimensions $L \times L$. Equation (19) implies that the width of an interface is determined by an intrinsic contribution $w_{0}$ that depends only on intensive variables and a term that explicitly depends on the area of the interface. For equilibrium systems, the stiffness coefficient $\kappa$ of an interface that separates two coexisting fluids is related to the surface tension via the simple relation $\gamma=k_{B} T_{s} \kappa$.

First, we test the applicability of Eq. (19) to our out-ofequilibrium system. To this end, we perform simulations with phase-separated systems of different sizes such that the area of the planar interface is set at $L^{2}, 2 L^{2}, 4 L^{2}, 9 L^{2}$, and $16 L^{2}$, with $L=14.7 \sigma$. The smaller area corresponds to the system of 2500 particles that we studied in Secs. IV A-IV C, while the bigger system has approximately 40000 particles. As we increase the system size, we find that the value of the surface tension reassuringly does not change, indicating that the results presented in Sec. IV C are free from large finite size effects. In order to measure the width of the interface, we first measure the density profile of the various systems. We find that as the system size is increased, Eq. (14) does not describe our simulation data as accurately as the error function fit,

$$
\rho(z)=\frac{1}{2}\left(\rho_{l}+\rho_{v}\right)-\frac{1}{2}\left(\rho_{l}-\rho_{v}\right) \operatorname{erf}\left[\frac{\sqrt{\pi}\left(z-z_{0}\right)}{w}\right],
$$

where the various parameters have the same interpretation as in Eq. (14). This observation has also been made for passive LJ systems. ${ }^{73}$ Thus, in this section, we use Eq. (20) in order to fit the density profiles $\rho(z)$ and estimate the width of the interface $w$ for different systems. 
We perform simulations for systems of different sizes for various combinations of the self-propulsion speed $v_{0}$, the rotational diffusion coefficient $D_{r}$, and the temperature $T$. Interestingly, we find that the width of the interface squared indeed scales linearly with the logarithm of the interfacial area, as Eq. (19) prescribes. In Fig. 5(a) we plot typical results for two sets of simulation parameters as well as the fit using Eq. (19). These fits allow us to extract the stiffness coefficient $\kappa$. Note that an equilibrium-like scaling of the width of the interface as a function of the interfacial area has previously been observed in the case of MIPS in a two-dimensional system. ${ }^{27}$

Next, we compare the value of the stiffness coefficient $\kappa$ as extracted from the scaling of the width of the interface with the area of the interface to the values of the surface tension $\gamma$ obtained by integrating the pressure tensor profiles [Eq. (15)] of the same system. The values of the two quantities have been acquired via independent measurements. Remarkably, we find that the two values can be related for all systems studied via the simple relation

$$
\begin{aligned}
\gamma & =\left(k_{B} T_{s}+\frac{\eta v_{0}^{2}}{6 D_{r}}\right) \kappa \\
& =k_{B} T_{\text {eff }} \kappa
\end{aligned}
$$

where we have defined an effective temperature $T_{\text {eff }}=T_{S}$ $+\eta v_{0}^{2} / 6 k_{B} D_{r}$. Note that this quantity has already been discussed in the literature as a means to connect active systems to their equilibrium counterparts; $; 3,8,9$ ideal passive particles with temperature $T_{\text {eff }}$ share on average the same translational diffusion rates as active Brownian particles with temperature
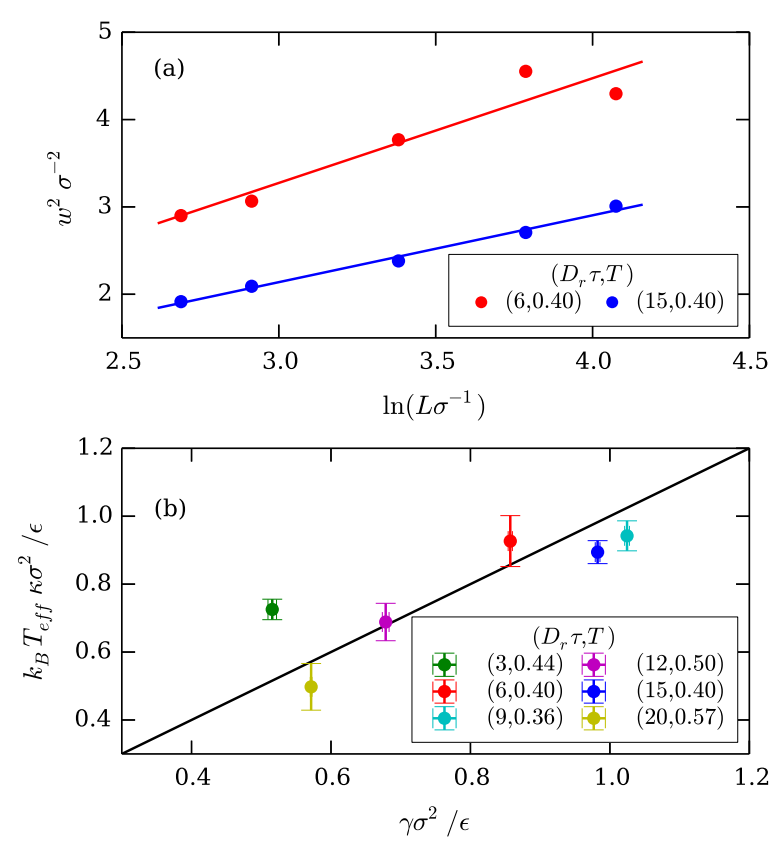

FIG. 5. (a) Interfacial width $w$ as a function of the length $L$ of the interface. Data points correspond to simulation results and the continuous lines are fits of Eq. (19). (b) The surface tension values measured from the stiffness coefficient $\boldsymbol{K}$ via Eq. (21) versus the interfacial tension as obtained via the pressure tensor route [Eq. (15)] for a range of different temperatures $T$ and rotational diffusion coefficients $D_{r} \tau$ as labeled. The black line shows the expected scaling $\gamma=k_{B} T_{\text {eff }} \kappa$. The speed of the self-propulsion is $v_{0} \tau \sigma^{-1}=8$ for all systems shown. Errors bars denote the error in the calculation of $\kappa$ from the fit of the width Eq. (19).
$T_{s}$, self-propulsion force $v_{0}$, and rotational diffusion $D_{r}$. In Fig. 5(b), we show the comparison between the scaled stiffness coefficient $k_{B} T_{\text {eff }} K$ as obtained from the scaling of the interfacial width and the surface tension $\gamma$ measured from the pressure tensor profiles for various system parameters. The figure confirms the applicability of Eq. (21) to our system, which we have further verified for various other system parameters (not shown here) and whose effective temperature $T_{\text {eff }} / T_{s}$ ranges from 1 to 100. As a final remark, note that Bialké et al. argued that a similar relation to Eq. (21) holds also in the case of MIPS, ${ }^{27}$ where $\gamma=-\kappa \eta v_{0}^{2} / D_{r}$ in two dimensions. However, an extra minus sign has to be included in this relation since the stiffness coefficient is positive while the surface tension is negative.

\section{CONCLUSIONS}

In conclusion, we performed Brownian dynamics simulations of a three-dimensional system of self-propelled particles interacting with Lennard-Jones interactions at state points that are well-inside the vapour-liquid phase coexistence region. We examine systems with a Péclet number $0 \leq v_{0} / D_{r} \sigma \leq 8$ so that we probe the equilibrium limit as well as systems that are out-of-equilibrium. However, in all cases, the phase separation is driven by the cohesive energy of the particles.

We studied the phase coexistence of a vapour and a liquid phase in an elongated simulation box and investigated the properties of the system and the interface. By employing a local expression of the pressure tensor for active systems, we measured the normal and tangential components of the pressure tensor in the direction perpendicular to the interface. We verified mechanical equilibrium of the two coexisting phases by measuring a constant normal component of the pressure tensor in the direction perpendicular to the interface. The tangential component showed negative peaks at the interface, behavior reminiscent of equilibrium systems and indicative of a positive non-equilibrium interfacial tension of the interface as measured by integrating the difference of the normal and tangential components of the pressure tensor.

We calculated the non-equilibrium surface tension for different combinations of self-propulsion speed and rotational diffusion rate and demonstrated that the trends of the surface tension can be fitted by simple power laws similar to equilibrium systems. These scaling laws enabled us to obtain an estimate for the critical temperature of the system as well. Interestingly, the resulting critical temperature of the active system was in close agreement with the values of the critical temperature obtained from the scaling of the order parameter. ${ }^{45}$ This agreement suggests on the one hand that the definitions of pressure and surface tension that were used constitute useful tools for the study of the physics of the phase transition and on the other hand hints to a deeper but not yet understood connection between the physics of the passive and the active systems.

Furthermore, we calculated the stiffness coefficient of the interface and found a simple equation that relates it to the surface tension. This relation had the same form as in equilibrium systems, assuming an effective temperature of the interfacial fluctuations. Our results show many similarities between bulk 
and interfacial properties of active and passive LennardJones systems for state points in the vapour-liquid coexistence region. We hope that by bringing these similarities into light, we inspire and assist theoretical work in the direction of building a statistical physics of active matter and its associated phase transitions.

\section{ACKNOWLEDGMENTS}

S.P. and M.D. acknowledge the funding from the Industrial Partnership Program "Computational Sciences for Energy Research" (Grant No. 14CSER020) of the Foundation for Fundamental Research on Matter (FOM), which is part of the Netherlands Organization for Scientific Research (NWO). This research programme is co-financed by Shell Global Solutions International B.V. V.P. and L.F. acknowledge funding from the Dutch Sector Plan Physics and Chemistry, and L.F. acknowledges financial support from the Netherlands Organization for Scientific Research (NWO-VENI Grant No. 680.47.432). We would also like to thank Berend v. d. Meer for careful reading of the manuscript and Robert Evans for useful discussions.

${ }^{1}$ V. Narayan, S. Ramaswamy, and N. Menon, Science 317, 105 (2007).

${ }^{2}$ R. Wittkowski, A. Tiribocchi, J. Stenhammar, R. J. Allen, D. Marenduzzo, and M. E. Cates, Nat. Commun. 5, 4351 (2014).

${ }^{3}$ S. C. Takatori and J. F. Brady, Soft Matter 11, 7920 (2015).

${ }^{4}$ U. Marini Bettolo Marconi and C. Maggi, Soft Matter 11, 8768 (2015).

${ }^{5}$ A. P. Solon, J. Stenhammar, R. Wittkowski, M. Kardar, Y. Kafri, M. E. Cates, and J. Tailleur, Phys. Rev. Lett. 114, 198301 (2015).

${ }^{6}$ G. Falasco, F. Baldovin, K. Kroy, and M. Baiesi, New J. Phys. 18, 093043 (2016).

${ }^{7}$ T. Speck and R. L. Jack, Phys. Rev. E 93, 062605 (2015).

${ }^{8}$ F. Ginot, I. Theurkauff, D. Levis, C. Ybert, L. Bocquet, L. Berthier, and

C. Cottin-Bizonne, Phys. Rev. X 5, 011004 (2015).

${ }^{9}$ T. Speck, Europhys. Lett. 114, 30006 (2016).

${ }^{10}$ S. Herminghaus and M. G. Mazza, Soft Matter 13, 898 (2017).

${ }^{11}$ J. P. Clewett, J. Wade, R. Bowley, S. Herminghaus, M. R. Swift, and M. G. Mazza, Sci. Rep. 6, 28726 (2016).

${ }^{12}$ J. P. Clewett, K. Roeller, R. M. Bowley, S. Herminghaus, and M. R. Swift, Phys. Rev. Lett. 109, 228002 (2012).

${ }^{13}$ A. Wysocki, R. G. Winkler, and G. Gompper, Europhys. Lett. 105, 48004 (2014).

${ }^{14}$ J. Stenhammar, D. Marenduzzo, R. J. Allen, and M. E. Cates, Soft Matter 10, 1489 (2014).

${ }^{15}$ J. Tailleur and M. E. Cates, Phys. Rev. Lett. 100, 218103 (2008).

${ }^{16}$ Y. Fily and M. C. Marchetti, Phys. Rev. Lett. 108, 235702 (2012).

${ }^{17}$ J. Bialké, H. Löwen, and T. Speck, Europhys. Lett. 103, 30008 (2013).

${ }^{18}$ C. F. Lee, Soft Matter 13, 376 (2017).

${ }^{19}$ T. F. F. Farage, P. Krinninger, and J. M. Brader, Phys. Rev. E 91, 042310 (2015).

${ }^{20}$ G. S. Redner, M. F. Hagan, and A. Baskaran, Phys. Rev. Lett. 110, 055701 (2012).

${ }^{21}$ G. S. Redner, A. Baskaran, and M. F. Hagan, Phys. Rev. E 88, 012305 (2013).

${ }^{22}$ M. E. Cates and J. Tailleur, Europhys. Lett. 101, 20010 (2013).

${ }^{23}$ J. Stenhammar, A. Tiribocchi, R. J. Allen, D. Marenduzzo, and M. E. Cates, Phys. Rev. Lett. 111, 145702 (2013).

${ }^{24}$ Y. Fily, S. Henkes, and M. C. Marchetti, Soft Matter 10, 2132 (2014).

${ }^{25}$ S. C. Takatori, W. Yan, and J. F. Brady, Phys. Rev. Lett. 113, 028103 (2014).

${ }^{26}$ A. Zöttl and H. Stark, Phys. Rev. Lett. 112, 118101 (2014).

${ }^{27}$ J. Bialké, J. T. Siebert, H. Löwen, and T. Speck, Phys. Rev. Lett. 115, 098301 (2015).
${ }^{28}$ S. C. Takatori and J. F. Brady, Phys. Rev. E 91, 032117 (2015).

${ }^{29}$ W. Yan and J. F. Brady, Soft Matter 11, 6235 (2015).

${ }^{30}$ H. C. Berg, E. coli in Motion (Springer, 2004).

${ }^{31}$ A. Czirók, E. Ben-Jacob, I. Cohen, and T. Vicsek, Phys. Rev. E 54, 1791 (1996).

${ }^{32}$ I. S. Aranson, C. R. Phys. 14, 518 (2013).

${ }^{33}$ J. Palacci, C. Cottin-Bizonne, C. Ybert, and L. Bocquet, Phys. Rev. Lett. 105, 088304 (2010).

${ }^{34}$ W. Gao, A. Uygun, and J. Wang, J. Am. Chem. Soc. 134, 897 (2012).

${ }^{35}$ A. A. Solovev, Y. Mei, E. B. Ureña, G. Huang, and O. G. Schmidt, Small 5, 1688 (2009).

${ }^{36}$ I. Buttinoni, J. Bialké, F. Kümmel, H. Löwen, C. Bechinger, and T. Speck, Phys. Rev. Lett. 110, 238301 (2013).

${ }^{37}$ G. Volpe, I. Buttinoni, D. Vogt, H.-J. Kümmerer, and C. Bechinger, Soft Matter 7, 8810 (2011).

${ }^{38}$ R. Golestanian, Phys. Rev. Lett. 108, 038303 (2012).

${ }^{39}$ M. M. Hanczyc, T. Toyota, T. Ikegami, N. Packard, and T. Sugawara, J. Am. Chem. Soc. 129, 9386 (2007).

${ }^{40}$ S. Thutupalli, R. Seemann, and S. Herminghaus, New J. Phys. 13, 073021 (2011).

${ }^{41}$ R. Dong, Q. Zhang, W. Gao, A. Pei, and B. Ren, ACS Nano 10, 839 (2016).

${ }^{42}$ J. Schwarz-Linek, C. Valeriani, A. Cacciuto, M. E. Cates, D. Marenduzzo, A. N. Morozov, and W. C. K. Poon, Proc. Natl. Acad. Sci. U. S. A. 109, 4052 (2012)

${ }^{43}$ B. M. Mognetti, A. Saric, S. Angioletti-Uberti, A. Cacciuto, C. Valeriani, and D. Frenkel, Phys. Rev. Lett. 111, 245702 (2013).

${ }^{44}$ V. Prymidis, H. Sielcken, and L. Filion, Soft Matter 11, 4158 (2015).

${ }^{45}$ V. Prymidis, S. Paliwal, M. Dijkstra, and L. Filion, J. Chem. Phys. 145, 124904 (2016).

${ }^{46}$ B. Smit, J. Chem. Phys. 96, 8639 (1992).

${ }^{47}$ J. Vrabec, G. K. Kedia, G. Fuchs, and H. Hasse, Mol. Phys. 104, 1509 (2006).

${ }^{48}$ H. Watanabe, N. Ito, and C.-K. Hu, J. Chem. Phys. 136, 204102 (2012).

${ }^{49}$ M. G. Noro and D. Frenkel, J. Chem. Phys. 113, 2941 (2000).

${ }^{50}$ D. Dunikov, S. Malyshenko, and V. Zhakhovskii, J. Chem. Phys. 115, 6623 (2001).

${ }^{51}$ R. G. Winkler, A. Wysocki, and G. Gompper, Soft Matter 11, 6680 (2015).

${ }^{52}$ A. P. Solon, Y. Fily, A. Baskaran, M. E. Cates, Y. Kafri, M. Kardar, and J. Tailleur, Nat. Phys. 11, 673 (2015).

${ }^{53}$ M. Dijkstra, S. Paliwal, J. Rodenburg, and R. van Roij, e-print arXiv:1609.02773 (2016).

${ }^{54}$ R. Evans, Adv. Phys. 28, 143 (1979).

${ }^{55}$ D. J. Higham and D. Higham, SIAM Rev. 43, 525 (2001).

${ }^{56}$ U. Marini Bettolo Marconi, C. Maggi, and S. Melchionna, Soft Matter 12, 5727 (2016).

${ }^{57}$ J. Rodenburg, M. Dijkstra, and R. van Roij, e-print arXiv:1609.08163 (2016).

${ }^{58}$ T. Ikeshoji, B. Hafskjold, and H. Furuholt, Mol. Simul. 29, 101 (2003).

${ }^{59}$ M. V. Berry, Phys. Educ. 6, 79 (1971).

${ }^{60}$ A. Marchand, J. H. Weijs, J. H. Snoeijer, and B. Andreotti, Am. J. Phys. 79, 999 (2011).

${ }^{61}$ A. Fortini, M. Dijkstra, M. Schmidt, and P. Wessels, Phys. Rev. E 71, 051403 (2005).

${ }^{62}$ P. Virnau and M. Müller, J. Chem. Phys. 120, 10925 (2004).

${ }^{63}$ K. Binder, Phys. Rev. A 25, 1699 (1982).

${ }^{64}$ J. Potoff and A. Panagiotopoulos, J. Chem. Phys. 112, 6411 (2000).

${ }^{65}$ M. Müller and L. MacDowell, J. Phys.: Condens. Matter 15, R609 (2003).

${ }^{66}$ S. W. Sides, G. S. Grest, and M.-D. Lacasse, Phys. Rev. E 60, 6708 (1999).

${ }^{67}$ M.-D. Lacasse, G. S. Grest, and A. J. Levine, Phys. Rev. Lett. 80, 309 (1998).

${ }^{68}$ F. P. Buff, R. A. Lovett, and F. H. Stillinger, Jr, Phys. Rev. Lett. 15, 621 (1965)

${ }^{69}$ D. Beysens and M. Robert, J. Chem. Phys. 87, 3056 (1987).

${ }^{70}$ M. A. Anisimov, Critical Phenomena in Liquids and Liquid Crystals (Gordon and Breach Science Publishers, 1991).

${ }^{71}$ W. Shi and J. K. Johnson, Fluid Phase Equilib. 187-188, 171 (2001).

${ }^{72} \mathrm{~J}$. Rowlinson and B. Widom, Molecular Theory of Capillarity (Dover Publications, 2002)

${ }^{73}$ A. E. Ismail, G. S. Grest, and M. J. Stevens, J. Chem. Phys. 125, 014702 (2006). 